\title{
Le linéaire B et l'administration des palais mycéniens
}

\author{
PASCAL DARCQUE \\ Directeur de Recherche, CNRS, \\ UMR Archéologies et Sciences de l'Antiquité, \\ Maison René Ginouvès, Nanterre
}

\begin{abstract}
RESUMO: Os tabletes de argila e os vasos que apresentam inscrições em escrita linear B se constituem numa documentação epigráfica relativamente restrita. Entretanto, o deciframento dessa escritura mostrou que se utilizava uma forma de grego em Creta e na Grécia continental seis séculos, ao menos, antes da época de Homero, e permitiu que se compreendessem documentos que são nossa principal fonte de informação sobre os palácios micênicos (fim do segundo milênio a.C.).
\end{abstract}

PALAVRAS-CHAVE: Grécia micênica; linear B; administração palaciana.

Les tablettes d'argile et les vases porteurs d'inscriptions rédigées en écriture linéaire B constituent une documentation épigraphique relativement restreinte, si on la compare à celle trouvée dans les palais mésopotamiens, levantins ou hittites. Cependant le déchiffrement du linéaire B en 1952, par M. Ventris, a constitué une véritable révolution dans les études concernant la protohistoire égéenne. Il a d'abord montré qu'on utilisait une forme de grec en Crète et en Grèce continentale six siècles au moins avant l'époque d'Homère. Il a également permis de comprendre des documents qui sont notre principale source d'information sur les palais mycéniens (fin du $\mathrm{II}^{\mathrm{e}}$ millénaire avant J.-C.).

\section{Historique des découvertes et du dechiffrement}

\section{a) Les découvertes}

De 1900 à nos jours, 7 sites ont livré des tablettes ou des nodules en argile crue portant des inscriptions en linéaire B.

En Crète, Knossos a livré plus de 3000 documents et La Canée 4 tablettes.

Sur le continent, Pylos, en Messénie (Sud-Ouest du Péloponnèse) a fourni 1100 documents, dont plus de 700 dans les seules pièces 6 et 7 de l'édifice principal. En Argolide, Mycènes a livré 73 documents, dont 63 proviennent des édifices fouillés au sud du cercle B (maison du Marchand d'huile, maison des Boucliers, maison des Sphinx et maison Ouest), Tirynthe 25 tablettes, dont 19 dans un édifice de la citadelle basse, et Midéa 3 nodules. À Thèbes, en Béotie, on a mis au jour plus de 300 documents, dont 56 nodules. 
Par ailleurs, on a découvert environ 160 vases à inscription peinte. La plupart de ces vases, des jarres à étrier d'une quarantaine de $\mathrm{cm}$ de haut, proviennent de sites qui ont livré d'autres documents inscrits en linéaire B : Thèbes (71 vases), Tirynthe (44), La Canée (28), Mycènes (12), Knossos (2) et Midéa (1). De plus, trois vases ont été découverts à Malia, ainsi que des exemplaires isolés à Orchomène, Gla, Kreusis, Éleusis, Arméni et Mamelouko.

\section{b) Le déchiffrement}

Le linéaire B a été déchiffré en 1952 par l'Anglais Michael Ventris. Trois conditions étaient alors remplies:

$1^{\circ}$ ) Ventris disposait d'un matériel d'étude quantitativement important : les tablettes découvertes à Knossos depuis 1900, ainsi que les 500 tablettes déjà découvertes à Pylos en 1939 ;

$2^{\circ}$ ) il avait mis au point et appliqué une méthode systématique de traitement analytique de ce matériel;

$3^{\circ}$ ) la langue que note le linéaire B était, même si Ventris ne s’en doutait pas au départ et qu'il a dû s'en convaincre lui-même par son propre déchiffrement, une langue connue - le grec - faisant partie d'un ensemble de langues connues et bien étudiées - les langues indo-européennes.

Le déchiffrement du linéaire B a été également rendu possible par les études auxquelles s'étaient livrés plusieurs chercheurs avant Ventris. A. Evans avait expliqué les notations numériques, E. Bennett avait mis en évidence le système des poids et mesures et analysé, de façon approfondie, les différentes catégories de signes (syllabogrammes et idéogrammes). A. Kober avait montré que certains groupes de signes apparaissaient avec trois terminaisons différentes, terminaisons recouvrant des genres ou des cas différents. Ventris, lui, est parvenu au déchiffrement en constituant des "grilles" où il ordonnait les syllabogrammes selon leurs valeurs vocaliques ou consonantiques supposées. Petit à petit sont apparus des squelettes de mots, en particulier des toponymes crétois (a-mi-ni-so, pour Amnisos, ko-no-so pour Knossos ou pa-i-to pour Phaistos) et des mots sonnant grecs. Ventris dut bientôt se rendre à l'évidence: la langue notée par le linéaire B est une forme de grec, ancêtre du groupe dialectal arcado-chypriote.

La preuve du déchiffrement, s'il en fallait une, est venue de la découverte, dans les fouilles du palais de Pylos, de la fameuse tablette dite "des trépieds" (PY Ta 641) - en mycénien $t i$-ri-po-de, grec $\tau$ pí $\pi 0 \delta \varepsilon \varsigma$-, laquelle devant des dessins (idéogrammes) de vases à trois anses fournissait aussi, écrit en toutes syllabes et en excellent grec, la glose "à trois anses" ( $t i-r i$-o-we), " à quatre anses " (qe-to-ro-we) devant des vases à quatre anses, et "sans anse" (a-no-we) devant le dessin d'un vase dépourvu d'anses.

\section{c) La forme des documents et la structure de l'écriture}

On distingue deux formes principales de tablettes. Les tablettes-pages sont, comme leur nom l'indique, plus hautes que larges, mais sur certaines le texte peut être disposé à l'italienne, c'est-à-dire parallèlement aux longs côtés; la plus grande connue, une tablette de Knossos (KN As 1516) mesure $27 \times 16$ × $3 \mathrm{~cm}$ et comporte 25 lignes d'écriture. Les tablettes-feuilles, longues et étroites, dont la forme a été comparée à celle des feuilles de palmier, portent la plupart du temps une ou deux lignes parallèles aux longs côtés. 
Le morceau d'argile ordinaire, parfois modelé autour d'un morceau de paille ou d'une baguette, est inscrit au moyen d'un stylet, puis simplement mis à sécher. C'est pourquoi seul un incendie a pu le conserver. Les tablettes étaient rangées dans des paniers ou dans des boîtes en bois, puis placées sur des étagères. On plaquait parfois une étiquette inscrite sur les paniers ou sur les boîtes, afin de les identifier.

Les nodules sont des petits morceaux d'argile de 1,5 ou $2 \mathrm{~cm}$ de côté, irrégulièrement façonnés autour d'une ficelle; ils comportent en général trois faces, deux éventuellement inscrites et une marquée d'une empreinte de sceau.

Le linéaire B comprend trois principales catégories de signes :

- des chiffres appartenant à un système décimal : 1, 10, 100, 1000 et 10.000 .

- une centaine d'idéogrammes symbolisant des êtres vivants $-* 100=$ VIR (homme), $* 102=$ MULIER $($ femme) $* 105=$ EQUUS $($ cheval $)-$, des objets $-* 162=$ TUNICA (cui rasse), $* 240=$ BIGAE (char), vases - , des poids et des mesures.

- 87 signes phonétiques notant des syllabes. Le système syllabique du linéaire $\mathrm{B}$ apparaît relativement peu complexe. Moyennant un nombre de conventions assez réduit, et inhérentes à tout syllabaire simple qui ne peut noter les groupes de consonnes autrement qu'en les décomposant - ko-no-so pour rendre Knos(s)os - ou en les réduisant à un seul de leurs éléments - pa-i-to pour Phaistos -, le linéaire B est, quoi qu'on ait pu en dire, un excellent outil pour écrire le grec. Il n'est certes pas aussi bien adapté que l'alphabet, mais il est incomparablement plus souple que les lourds systèmes égyptien ou mésopotamien. Même la notation des " choses " recensées, souvent représentées de façon réaliste, se révèle très aisée. De plus, il s'agit d'un outil de caractère homogène et universel, uniforme de la Béotie au Nord jusqu'à la Crète au Sud, reflétant partout un même état de langue, le grec des administrateurs des palais mycéniens. Les différences très minimes que l'on y décèle ne sont rien à côté de celles que l'on peut trouver entre les différents alphabets grecs archaïques.

\section{La date des documents et l'origine du linéaire B}

D'après les documents archéologiques dont nous disposons actuellement, l'usage du linéaire B est attesté durant une période de 200 ans. Les plus anciens documents inscrits en linéaire B datent des environs de 1400 avant J.-C. Ils ont été découverts à Knossos. Ils se situent 120-150 ans avant les "premières" tablettes continentales, celles de Pylos ou de la ville basse de Mycènes (vers 1250 avant J.-C.), et 200 ans avant les documents les plus récents (tablettes de Tirynthe dans un niveau de destruction des environs de 1200; tablettes de la citadelle de Mycènes).

La date des tablettes de Knossos a fait l'objet de débats passionnés. Les recherches récentes montrent que le site de Knossos a subi quatre ou cinq destructions entre 1400 et 1200; plusieurs ensembles de tablettes correspondraient à chacune de ces destructions.

L'homogénéité de contenu et de langue autorise à considérer les documents inscrits en linéaire B comme une source cohérente sur une institution - le palais - qu'on imagine aisément conservatrice. Mais on ne doit jamais oublier que ces documents reflètent des situations chronologiquement et géographiquement distinctes.

Le linéaire $\mathrm{B}$ a été créé à partir d'une forme du linéaire $\mathrm{A}$, relativement archaïque, un linéaire A écrit sur matériau souple, avec un instrument flexible. Cette forme n'est pas directement attestée, mais ne devait pas être fondamentalement différente de celles que 
nous connaissons. En effet, sur 87 syllabogrammes du linéaire B, 64, qui sont en général les plus fréquents, ont un ancêtre pratiquement assuré en linéaire A et 23 seulement semblent bien être des créations originales. Si la filiation linéaire A-linéaire B est admise par tous les chercheurs, l'origine même du linéaire B reste une question très controversée. Pour les uns, le linéaire B aurait été créé en Grèce continentale, sans doute en Argolide, dès le début de l'époque mycénienne. Pour les autres, l'unité graphique des documents continentaux ne peut s'expliquer que par un emprunt à une source unique, crétoise.

La date de cette création reste très difficile à fixer. Avant 1500, l'état de développement de la civilisation mycénienne ne paraît pas justifier l'acquisition et l'emploi régulier d'une écriture. Mais le premier linéaire B attesté, qui date de 1400, possède déjà une "épaisseur ", linguistique et paléographique, telle qu'il faut lui supposer une existence déjà longue au moment où il apparaît.

\section{Le fonctionnement des palais mycéniens}

Les tablettes mycéniennes se distinguent nettement des documents épigraphiques retrouvés sur les sites anatoliens, levantins ou mésopotamiens et rédigés en sumérien, égyptien, accadien, hourrite, hittite ou ougaritique. On n'y trouve en effet ni documents commerciaux, ni archives administratives ou juridiques, ni correspondance diplomatique, ni écrits relatifs à la vie religieuse ou intellectuelle, ni œuvres littéraires.

De nombreux chercheurs estiment que ces documents étaient inscrits sur des matériaux périssables, comme le papyrus et la peau d'animal. En outre, les tablettes que nous possédons datent seulement de la dernière année de fonctionnement des palais. Certaines d'entre elles font allusion à des comptes antérieurs. On serait donc en droit de considérer les documents qui nous sont parvenus non comme de véritables archives, mais comme des "brouillons d'archives", suivant l'heureuse expression de J.-P. Olivier', et de supposer qu'il nous manque l'essentiel. Ne peut-on pas cependant risquer l'hypothèse que ce qui nous a été livré, en particulier, par les fouilles de Pylos représente assez fidèlement ce qui y était conservé? La répartition des rations alimentaires ou les prélèvements fiscaux suivent des règles qui devaient être transmises d'une génération à l'autre d'administrateurs, de même que les comptables modernes, publics ou privés, connaissent parfaitement la structure de leur plan comptable, sans avoir besoin de se référer aux comptes de l'année précédente. De même, les lignes créditrices ou débitrices sont naturellement intégrées à la comptabilité de l'année en cours. On pourrait supposer que les administrateurs se contentaient d'actualiser la comptabilité de l'année précédente en se référant, d'une part, à leur "plan comptable" (règles de taxation et de répartition des produits, ordre de citation), et, d'autre part, aux tablettes en terre crue conservées d'une année sur l'autre. Celles-ci seraient alors effacées, comme le montrent les palimpsestes, ou complètement remodelées par les scribes eux-mêmes ou des aides. Nous pourrions alors parler de véritables archives, comme nous y invite, par ailleurs, la structure des documents conservés dans les pièces 7 et 8 de Pylos.

Comme le travail des scribes n'était pas de décrire l'administration des palais, mais d'enregistrer des inventaires de magasins, des productions agricoles, des contributions, des répartitions de matière première, des divisions de terre ou des distributions de vivres, l'interprétation historique des documents reste souvent difficile. Ils n'en demeurent pas moins une des sources essentielles sur la vie économique et sociale du monde mycénien. 
Les textes disponibles jettent une lumière assez précise sur certains aspects : l'organisation de la société palatiale, la structure des territoires dépendants, le rôle joué par les palais dans certains domaines de l'agriculture et de l'artisanat.

\section{a) L'organisation de la société}

Dans les documents dont nous disposons, 117 termes désignent des professions, des fonctions ou des dignités. Même pour les 40 termes qui trouvent leur équivalent dans le grec du $\mathrm{I}^{\text {er }}$ millénaire, de nombreux débats opposent les spécialistes afin de déterminer la réalité des pouvoirs, des statuts ou des activités que recouvrent ces termes.

Ainsi se demande-t-on si le mot WA-NA-KA désigne un roi humain, un souverain divin ou même seulement un dignitaire local. Le terme, et ses dérivés, assimilé dès les premiers temps du déchiffrement au ơv $\alpha \xi \xi$ homérique, apparaît sur quatre vases inscrits et sur une quarantaine de tablettes.

Dans un grand nombre de textes, le wa-na-ka peut passer pour un souverain humain. Il détient une autorité qui se manifeste par la nomination ou la mutation de fonctionnaires. Il serait le maître unique du palais et du royaume, car on néglige de préciser son nom. Ce pouvoir s'accompagne de privilèges matériels : le wa-na-ka possède un domaine; des artisans travaillent pour son service ou pour le service de son domaine; les scribes distinguent nettement et fréquemment ce qui est wa-na-ka-te-ro - "royal", appartenant au "roi", revenant au "roi" - de ce qui ne l'est pas. Mais, tandis que le ǒv $\alpha \xi$ homérique peut être relativement bien caractérisé, la réalité exacte que recouvre le terme wa-na-ka n'apparaît pas en toute clarté. L'hérédité de la fonction, la nature et les attributs de l'autorité, l'étendue réelle du domaine restent des questions en suspens. La traduction même du terme par le mot "roi" semble aller au-delà de ce que permettent les textes. A Pylos, par exemple, sur près d'un millier d'artisans mentionnés dans les tablettes, seuls trois sont qualifiés de wa-na-ka-te-ro. Qu'en conclure sur le statut des autres? Le secteur prétendu "royal" ne représenterait-il qu'une petite partie de l'activité contrôlée par le palais ? Si tel est le cas, est-on encore autorisé à traduire wa-na-ka par "roi", en supposant que ce titre désigne le maître unique du palais?

De la même façon, le rapprochement entre le terme mycénien $Q A-S I-R E-U$ et le grec $\beta \alpha \sigma \imath \lambda \varepsilon v \zeta$ a soulevé bien des difficultés quand on a voulu, tant bien que mal, identifier les fonctions recouvertes par ces mots. Chez Homère, en effet, le mot $\beta \alpha \sigma \imath \lambda \varepsilon v ́ \varsigma$ désigne des rois ou des membres du conseil. La condition des qa-si-re-we connus par les textes mycéniens paraît, elle, très variable: ils peuvent être des "chefs", en particulier de groupes de forgerons, des dignitaires provinciaux ou de simples fonctionnaires locaux. Ils possèdent un domaine, mais doivent allégeance au wa-na-ka. Leur nom n'est pas associé aux capitales des royaumes, mais à des localités provinciales.

\section{b) Le fonctionnement de l'administration}

Un des progrès majeurs dans la connaissance de l'administration mycénienne a été accompli, durant les trente dernières années, grâce à des études très précises, œuvres de J.P. Olivier et Th. G. Palaima, consacrées à la structure des archives et à l'identification des scribes. On estime aujourd'hui que les quelque 3000 tablettes de Knossos sont dues à une centaine de scribes et les 1100 documents de Pylos à une cinquantaine d'individus différents. Certaines mains de scribe ne sont attestées qu'une seule fois, mais 240 tablettes sont 
attribuées au scribe $\mathrm{n}^{\circ} 1$ de Pylos, un archiviste qui récapitule le contenu de certains dossiers et intervient sur des tablettes écrites par d'autres. Le grand nombre de scribes relativement à la quantité de documents connus, leur spécialisation fait penser que les scribes ne sont pas, comme au Proche-Orient, des écrivains professionnels, mais des administrateurs lettrés qui se font peut-être aider pour la fabrication des tablettes. On ne connaît aucun mot désignant celui qui écrit, mais cette lacune pourrait n'être que provisoire.

Il n'existe de véritable salle d'archives qu'à Pylos où l'on a découvert plus de 700 tablettes groupées dans les pièces 7 et 8 . Tous les types d'enregistrements y sont représentés : fiches individuelles, recensements, récapitulatifs. Les "bureaux spécialisés" dans une branche de l'économie, par exemple l'élevage des ovins et l'industrie textile, semblent eux plus caractéristiques de l'organisation de Knossos. A Pylos et à Thèbes, on connaît également de simples dépôts enregistrant l'activité d'un magasin ou d'un atelier.

Les 56 nodules inscrits découverts à Thèbes en 1982 ont mis en évidence l'une des procédures de l'administration mycénienne. On a pu montrer en effet que ces nodules avaient accompagné des animaux (ovins, caprins, porcins et bovins) envoyés de toute la Béotie, et parfois de l'Eubée, vers le palais de Thèbes. Ces nodules ont joué le rôle de certificats de provenance et d'authenticité. Ils servaient ensuite à un scribe pour établir une tablette récapitulant l'ensemble de l'opération de transfert.

Les dimensions de ces archives sur argile et ce qu'on peut appréhender de l'organisation bureaucratique ne correspondent pas au fonctionnement d'une administration omnisciente et omniprésente. Même si la connaissance du linéaire B a dépassé le strict cadre palatial, comme semblent le montrer les nodules de Thèbes, on est frappé de l'usage limité qui en est attesté : moins de 200 jarres à étrier portent une inscription, alors qu'il en a circulé des milliers dans le monde égéen et en Méditerranée; on n'a découvert quasiment aucun graffiti en linéaire B; pour l'instant, on ne connaît qu'une seule inscription sur un sceau.

Il faut également souligner la disparition totale de cette écriture après 1200.

\section{c) La structure des territoires dépendants}

Les tablettes de Pylos montrent que le territoire dépendant du palais est divisé en deux provinces, elles-mêmes subdivisées en un certain nombre de districts. Les deux provinces du territoire administré par Pylos se situent de part et d'autre de la montagne appelée par Strabon (VIII, 359) Aigaléon. La "province proche" englobe la plaine côtière. Pylos pu-ro en mycénien -, capitale de l'État, en est le centre sans en constituer un des neuf districts. La "province lointaine" est constituée de sept districts essentiellement, mais non exclusivement continentaux. La capitale provinciale s'appelle re-u-ko-to-ro. Les noms des chefs-lieux des seize districts que comportent les deux provinces apparaissent sur divers documents concordant sur l'essentiel. La localisation même de chaque chef-lieu, la délimitation des districts, des deux provinces et du royaume, de même que la non-coïncidence des sources épigraphiques et du Catalogue des vaisseaux homérique, demeurent des questions vivement controversées.

Rien n'établit formellement, dans l'état actuel des recherches, que le "modèle " d'organisation attesté à Pylos prévalait dans toutes les régions de la Grèce continentale; la situation géographique et l'évolution historique de certains sites ont pu leur assurer un statut indépendant des grands royaumes. 
L'image, fournie par les tablettes en linéaire B, d'une stratification sociale et d'une organisation politique fonctionnant non pas tant au service d'un individu que d'un groupe dominant ne vaut que pour les territoires "dépendants" de Mycènes, Tirynthe, Pylos et Thèbes. Il reste difficile de l'étendre à des régions qui, comme l'Arcadie, la Laconie, l'Élide ou la Phocide, semblent relativement marginales. Cela vaut aussi pour l'organisation économique aperçue dans les documents en linéaire B.

\section{d) Le rôle économique du palais}

Les économies mycéniennes ne sont sans doute pas entièrement dépendantes du palais, mais le palais fait produire une part importante des richesses de chaque État ou procède à des prélèvements sur les productions. Grâce aux comptes tenus de ces prélèvements et des redistributions, nous apercevons le rôle économique joué par le palais, en particulier dans certains domaines de l'agriculture et de l'artisanat. Cela peut nous conduire à surestimer sa place dans le fonctionnement économique, car nous ne possédons d'informations que sur lui.

Les oléagineux. Les Mycéniens connaissent une série de plantes oléagineuses à partir desquelles pouvaient être obtenues des huiles alimentaires ou industrielles : sésame, lin, et sans doute d'autres, comme le ricin, le safran et le pavot. Mais la place de ces oléagineux reste très secondaire par rapport à celle de l'olivier.

Dans les archives de Knossos et de Pylos, les plus grandes quantités d'huile ne paraissent pas destinées à l'alimentation. L'usage industriel est partout nettement prédominant. On connaît plusieurs noms d'huile parfumées. Un texte de Pylos (PY Un 267), qui semble donner une "recette" de parfum incluant coriandre, cypérus, fruits, vin, et miel, indique par les quantités mentionnées - de $6 \mathrm{~kg}$ à 720 litres - et par la désignation d'un "bouilleur d'onguent " que nous sommes en présence d'une activité industrielle nécessitant l'emploi d'une main-d'œuvre spécialisée.

Cette huile parfumée était stockée et transportée dans des jarres à étrier, le type de vase que l'on trouve le plus fréquemment exporté en dehors de la Grèce mycénienne.

La métallurgie. L'organisation de la métallurgie est aperçue à travers la série Jn de Pylos. Au moins 400 forgerons, dispersés dans plus de 25 localités, sont recensés et le palais distribue une tonne de bronze à une partie d'entre eux. Comme l'allocation moyenne se monte à $3,5 \mathrm{~kg}$, on peut supposer que le travail demandé sur cette petite quantité de métal n'occupait pas complètement les artisans. Ceux-ci, d'autre part, ne figurent pas sur les listes de rationnaires; leur statut pourrait donc être relativement indépendant. En certaines occasions, le palais est amené à demander aux communautés ou individus placés sous son autorité des contributions en bronze et en or; cela pourrait signifier qu'il ne contrôle pas totalement la circulation de ces matières premières.

L'industrie textile. La production des textiles parait avoir été une des activités majeures des palais mycéniens. Les traces que nous en trouvons conservées dans les tablettes justifient même qu'on parle d'une véritable industrie. Ainsi près de 1000 tablettes de Knossos, sur 3 000, concernent les ovins, dont le nombre peut être estimé à près de 100000. On peut estimer la production annuelle moyenne des troupeaux de la série D de Knossos à 
une cinquantaine de tonnes de laine. Si l'on évalue le poids moyen des pièces de tissu à $10 \mathrm{~kg}$, ces 50 tonnes devaient permettre de produire environ 5000 pièces. Or les tablettes de la série L, qui concernent le travail de la laine de l'année précédente, recensent quelque 5000 pièces de tissu dont le poids unitaire varie entre 5 et $60 \mathrm{~kg}$ et le poids total atteint 45 tonnes. Les concordances entre ces deux séries permettent de supposer que la production ne variait pas beaucoup d'une année sur l'autre.

L'industrie lainière est également attestée à Pylos, mais de façon beaucoup plus fragmentaire.

A Pylos comme à Knossos, la majorité des ouvrières recensées par le palais travaillent le lin et la laine. Au moins quinze spécialités sont mentionnées dans les tablettes de Pylos, ce qui suggère une division extrême du travail conforme aux exigences d'une véritable “ industrie ". La série Lc de Knossos indique que dix tonnes de laine sont réparties entre une trentaine d'ateliers où travaillent 600 à 900 ouvrières.

Paradoxalement, nous avons davantage de renseignements sur les activités productrices dont il ne subsiste quasiment aucune trace archéologique, comme la fabrication des tissus et des parfums, que sur les autres. Ainsi, malgré les énormes quantités de céramique retrouvées sur certains sites, par exemple dans le palais de Pylos qui a livré plus de 7000 vases complets de 80 formes différentes, seuls quatre potiers (ke-ra-me-we) apparaissent dans les archives.

Par ses prélèvements et par les productions issues de ses propres terres ou ateliers, le palais emmagasine un grand nombre de produits. Certaines denrées sont redistribuées à une population plus ou moins dépendante sous forme de rations alimentaires, de matières premières ou de produits finis (vêtements, armes). Tout n'est sans doute pas redistribué, car le centre palatial doit dégager des surplus échangeables contre les produits ou matières premières dont il ne dispose pas. Les modalités de ces échanges entre les différents centres ou avec des régions extérieures au continent grec sont très mal connues. Les témoignages épigraphiques nous apprennent seulement que des tissus et de l'huile étaient spécialement fabriqués pour être exportés.

Par sa capacité d'organiser des productions spécialisées, de stocker et de redistribuer les produits, le système palatial mycénien s'apparente d'assez près aux systèmes contemporains ou plus anciens connus au Proche-Orient. Aussi longtemps que les centres palatiaux mycéniens fonctionnent, leur pouvoir économique et politique apparaît relativement fort et centralisé. Mais, s'il faut en croire les tablettes de Pylos, ce pouvoir ne semble pas avoir dépassé un cadre strictement régional. Et, même dans ce cadre, il ne faut pas assimiler l'activité économique du palais à celle de la société dans son ensemble.

Comme en Orient et comme en Crète, l'administration d'une population et d'un territoire dépendants s'inscrit dans un cadre architectural stéréotypé, l'édifice qu'on appelle “palais ". La disparition des palais mycéniens, entre 1250 et 1200 avant J.-C., entraîne la disparition du système palatial dans le monde égéen. En même temps on cesse d'utiliser le linéaire B. Ce bouleversement politique et culturel ouvre la voie à un nouveau mode d'organisation économique, politique et sociale, à une nouvelle structuration de l'espace égéen, en cités, et à une nouvelle écriture, le grec alphabétique.

\section{Note}

1- Les Dossiers d'Archéologie 195 (juillet-août 1994), p. 56. 


\section{Bibliographie}

CHADWICK, J. Le déchiffrement du linéaire B. Aux origines de la langue grecque. Paris: Gallimard, 1972.

MIRO, E. DE, GODART, L \& SACCONI, A (Éd.). Atti e Memorie del Secondo Congresso Internazionale di Micenologia, Roma-Napoli, 14-20 ottobre 1991, Incunabula Graeca 98 Rome: Gruppo Editoriale Internazionale, 1996.

GODART, L. Le pouvoir de l'écrit. Aux pays des premières écritures. Paris: Éditions Errance, 1990 (Collection des Néréides).

MORPURGO DAVIES, A. \& DUHOUX, Y (Éd.). Linear B: a 1984 survey. Proceedings of the Mycenaean Colloquium of the VIIIth Congress of the International Federation of the Societies of Classical Studies (Dublin, 27 August - 1st September 1984). Bibliothèque des Cahiers de l'Institut de Linguistique de Louvain 26, Cabay, Louvain-la-Neuve, 1985.

OLIVIER, J.-P. (Éd.). Mykenaïka. Actes du IXe Colloque International sur les Textes Mycéniens et Égéens (Athènes, 2-6 octobre 1990), Bulletin de Correspondance hellénique. Supplément XXV, École Française d'Athènes, 1992.

SHELMERDINE, C. Review of Aegean Prehistory VI: The Palatial Bronze Age of the Southern and Central Greek Mainland. American Journal of Archaeology 101, p. 537$585,1997$.

TREUIL, R. et alii. Les civilisations égéennes du Néolithique et de l'Age du Bronze. Paris: Presses Universitaires de France, 1989.

VENTRIS, M. \& CHADWICK, J. Documents in Mycenaean Greek, $2^{\mathrm{e}}$ éd. Cambridge: Cambridge University Press, 1973.

DARCQUE, Pascal. O linear B e a administração dos palácios micênicos. Classica, São Paulo, 11/12, p. 139-147, 1998/1999.

RÉSUMÉ: Les tablettes d'argile et les vases porteurs d'inscriptions rédigées en écriture linéaire $B$ constituent une documentation épigraphique relativement restreinte. Cependant le déchiffrement de cette écriture a montré qu'on utilisait une forme de grec en Crète et en Grèce continentale six siècles au moins avant l'époque d'Homère. II a également permis de comprendre des documents qui sont notre principale source d'information sur les palais mycéniens (fin du $I^{e}$ millénaire avant J.-C.).

MOTS-CLÉS: Grèce mycénienne; linéaire B; administration palatiale. 